\title{
Per totam linguaginem
}

\author{
['In every language']
}

\author{
PÄr LARSON \\ Istituto Opera del Vocabolario Italiano (OVI-CNR)
}

ubi tunc (...) vox inaudite lingue?

Volmar $(† 1173)$

Sommario. L'articolo è una rivisitazione di un testo mediolatino compostellano del XIII secolo trasmesso da una copia vergata nel 1328, resa nota al pubblico da A. López Ferreiro nel 1902 e studiata da J. L. Pensado nel 1960. L'autore propone una nuova analisi delle quattro brevi frasi in lingue diverse dal latino inserite nel testo, concentrandosi in particolare sull'ultima di queste frasi, la quale, sebbene scritta in una lingua sconosciuta, sembra tuttavia avere un significato diverso dalle fantasiose interpretazioni proposte dagli studiosi che l'hanno affrontata nell'ultimo secolo e mezzo. Parole chiave: Santiago di Compostella nel medioevo, pellegrinaggi, conoscenza di lingue straniere nel medioevo.

Abstract. The author revisits a thirteenth-century medieval Latin text from Santiago de Compostela preserved as a copy from 1328 , which was made known to the reading public by A López Ferreiro in 1902 and studied by J. L. Pensado in 1960. The paper provides a new analysis of the four short phrases in languages other than Latin inserted in the text; in particular, it focuses on the last of these phrases, written in an unknown language, which nevertheless seems to yield

Data de recepción: 21-09-2019 - Data de aceptación: 27-12-2019. 
a meaning different from the fanciful interpretations proposed by the scholars who dealt with it during the last one and a half centuries.

Keywords: Medieval Santiago de Compostela, pilgrimage, knowledge of foreign languages during the Middle Ages.

IN UN CODICE MEMBRANACEO vergato nel 1328 e custodito nell'Archivio della Cattedrale di Santiago de Compostela è copiato un testo latino databile alla metà del secolo $\mathrm{XIII}^{1}$, che fornisce informazioni preziose dal punto di vista storico sulle mansioni dei chierici e degli ufficiali laici della Cattedrale, nonché sulle abitudini dei pellegrini che vi concludevano il loro iter. Ancor più prezioso si rivela il nostro testo dal punto di vista linguistico, sia per il tipo di latino in cui fu redatto, sia per le frasi in diverse lingue che contiene.

Le Consuetudines quas custodes Arche Operis Beati Jacobi consuevit observare cum custodibus Altaris Beati Jacobi - così recita la rubrica ${ }^{2}$ — sono il risultato di un'inchiesta ordinata dall'arcivescovo compostellano, come si apprende dalla sottoscrizione dello scriba, che dichiara di avere agito su richiesta del cantor Joan Paez, del cardinalis Lourenço Dominguez e di un certo Joan Fernandez detto «Rapaz», qui hec de mandato Domini Archiepiscopi inquisierunt ${ }^{3}$.

Le Consuetudines furono pubblicate per la prima volta dal benemerito canonico Antonio López Ferreiro nel quinto tomo della sua Historia de la Santa Apostólica y

Santiago de Compostela, Arquivo da Catedral, Constituciones de la Iglesia de Santiago, vol. $2^{\circ}$, ff. $64 r$ - $65 r$. Il codice, che porta la segnatura CF20, misura $\mathrm{cm} 37,5 \times 26$ ed è composto da 91 fogli (ringrazio Michele Colombo del controllo finale del ms. per conto mio). Il documento originale contenente le Consuetudines fu vergato da Johannes Pelagii, publicus notarius Compostellanus juratus, probabilmente da identificarsi con l'omonimo notaio che sottoscrive, usando la medesima formula, tre documenti del cartulario del monastero di Toxos Outos negli anni 1245, 1248 e 1255: cfr. Pérez Rodríguez (2004: 387, 411 e 419 [docs. 388, 417 e 426], nel secondo di questi compare anche «Johannes Fernandi dictus Rapatus», per cui vedi infra, nota 3).

2 Questa è preceduta da un'altra, formulata secondo il punto di vista dei canonici legati all'altare dell'Apostolo, e quindi con ogni probabilità aggiunta al momento della copiatura delle Consuetudines nel manoscritto: Qualiter Custodes Altaris Beati Jacobi debent se habere erga custodes Operis Beati Jacobi et etiam de alijs honoribus Ecclesie Sancti Jacobi 'In qual modo i custodi dell'Altare del Beato Jacopo si devono comportare con i custodi dell'Opera del Beato Jacopo, e anche degli altri onori della Chiesa di San Jacopo'.

3 «Ego Johannes Pelagii publicus notarius Compostellanus juratus scripsi de mandato Johannis Pelagii cantoris, et Laurentij Dominici Cardinalis Compostellani et Johannis Fernandi dicti Rapati, qui hec de mandato dominj Archiepiscopi inquisierunt» (rr. 63-65). 
Metropolitana Iglesia de Santiago de Compostela, del 1902 (López Ferreiro 1902, Apéndice: 64-67); una nuova edizione, nelle intenzioni (un po' meno nei risultati) migliorativa rispetto a quella di López Ferreiro, venne inclusa in un'opera collettiva sul tema dei pellegrinaggi compostellani diretta dallo storico Luis Vázquez de Parga e pubblicata nel 1948-1949 (Vázquez de Parga et alii 1948-49, III: 113-114). Finalmente, nel 1960, l'accurata trascrizione del filologo galego José Luis Pensado, accompagnata da una riproduzione fotografica e da una dettagliata (anche se non irreprensibile) analisi linguistica, pose le basi per uno studio serio del documento.

Leggiamo il testo, avvertendo (1) che il brano qui riprodotto corrisponde a un po' meno di un terzo del testo complessivo ${ }^{4}, \mathrm{e}(2)$ che gli honores più volte menzionati sono le reliquie, gli altari e altri oggetti o luoghi della Cattedrale collegati con determinate indulgenze e perciò costantemente frequentati dai pellegrini. Giova infine tenere in mente che cos'era la «Arca dell'Opera», menzionata ben trentacinque volte nel testo complessivo:

Delante de la imagen de Santiago Alfeo, contigua al actual púlpito del Evangelio, había entonces un altar, y al lado una gran arca en que se recogían las limosnas y ofrendas que venian para la Fábrica de la Iglesia. (...) Todo lo que se recogía en esta arca era para los oficiales de la obra; los cuales per esto pagaban ciertos censos al Cabildo (López Ferreiro 1902: 255).

$$
* * *
$$

Hec sunt consuetudines, ${ }^{2} \mid$ quas custodes Arche Operis Beati Jacobi consuevit observare cum custodibus Altaris ${ }^{3} \mid$ Beati Jacobi. Primo, quam cito pulsata fuerit campana in Altare Beati Jacobi ad missam ${ }^{4} \mid$ matutinalem, arqueyrus ${ }^{5}$ (sive custos Arche) et clericus debent stare ibi ad Archam Operis cum suis ${ }^{5}$ varis in manu ad vocandum $\mathrm{p}$ [er] egrinos ad Archam et ad dandum cum eis in tergis et in membris ${ }^{6}$ peregrinorum loco primarie. Sed in Gradecellis portarum et portis Altaris Beati Jacobi nec alibi ${ }^{7}$ non debent cum eis dare ad faciendum cum eis strepitum ${ }^{6}$ vel tumultum.

$4 \quad$ Le abbreviazioni sono sciolte in corsivo (eccezion fatta per il quarto inserto alloglotto, riprodotto senza sciogliere i compendi) con l'introduzione di caratteri maiuscoli e minuscoli e distinzione tra $\langle\mathrm{u}\rangle \mathrm{e}\langle\mathrm{v}\rangle$. La continuazione del testo, per la quale rinvio a Pensado (1960), non aggiunge ulteriori informazioni sui fatti studiati e discussi nel presente articolo.

5 Il titolo di questo ufficiale della Cattedrale, sempre scritto arqueýrus tranne per un'occorrenza della forma più latina archarius, non va confuso con il galego-portoghese arqueiro 'arciere'.

$6 \quad$ Ms. scriptū. Pensado (1960: 328) pensava a una latinizzazione dell'occitano escrit 'grido', ma il binomio strepitum - tumultum è formulare in latino, soprattutto giuridico, fin dalla tarda antichità. 
Et clericus debet se $^{8} \mid$ vestire superpelicium suum et stare super Archa, et ille qui dixerit indulgencia $m$ sive perdone $m$ debet ${ }^{9} \mid$ primo Archam nominare ante quam alium honorem ecclesie. Et ex quo fuerit nominata, statim ipse ${ }^{10} \mid$ arqueyrus debet dicere Francigenis:

ze é l'archa de l'obra mon señor San Jame, ze é l'obra ${ }^{11}$ dela egresa.

Et Lombardis et Tozcanis debet dicere:

$\mathrm{O}$ miçer lombardo, quest'è l'archa dela ${ }^{12} \mid$ lavoree de micer Sa' Jacome. Questo vay ala gage fayr.

Et Campisinis debet dicere: ${ }^{13}$

Et vos et del estremo ${ }^{7}$, acá venide ala archa dela obra de Señor Santiago: las come $n^{14} \mid$ das que trahedes de mortos et de vivos para la obra de Señor Santiago acá las echa ${ }^{15} \mid$ de $e t$ non en outra parte.

Et unaquaque istarum parabolarum debet dici semel mane ex quo ${ }^{16} \mid$ Archa fuerit nominata in perdone et non ante, et exinde ipse et alii de ecclesia debent stare ${ }^{17}$ taciti quousque indulgencia dicatur. Et perdone dicto, debent vocare totum peregrinum $\mathrm{ad}^{18} \mid$ Archam per totam linguaginem et debet dicere:

btom aatrō sangyama aatrō d'labro. ${ }^{19}$

Et exinde matinata exita quando compania peregrinorum yverit per ad Altare Sancti $\mathrm{Jaco}^{20} \mid \mathrm{bi}$, debet arqueyrus vel homo eius quem ibi tenuerit nominare eis Archam, et si intellexerit quod peregrinus ${ }^{21} \mid$ vellit ibi mittere oblationem Altaris Beati Jacobi, debet sibi dicere et mostrare Altare Beati ${ }^{22}$ Jacobi, et dicere quod illa est Archa Operis. Et debent peregrini ita guiari videlicet quod primitus ${ }^{23}$ offerant Altari Beati Jacobi et exinde Cathene et exinde Arche Operis et exinde aliis ${ }^{24}$ | honoribus.

\section{TRADUZIONE}

Per prima cosa, appena la campana dell'Altare del Beato Jacopo avrà chiamato i fedeli alla messa del mattino, l'arqueiro (ossia il custode dell'Arca) e un chierico devono trovarsi accanto all'Arca dell'Opera con le loro pertiche in mano per

López Ferreiro (1902: 65) e Vázquez de Parga et alii (1948-49 [t. III]: 113) stampano «Et uos de Campos et del estremo», senza segnalare in alcun modo l'integrazione operata. 
chiamare i pellegrini all'Arca e per prima cosa colpirli sulla schiena e sulle membra. All' 'inferriata delle porte e alle stesse porte dell'Altare del Beato Jacopo e altrove non devono invece usare le pertiche, dato che potrebbero causare disordine e rumore. E il chierico deve indossare il suo camice da messa e stare in piedi sopra l'Arca, e colui che pronuncerà la formula dell'indulgenza (ossia il perdono) deve menzionare l'Arca prima di ogni altro onore della chiesa. E dopo che sarà stata menzionata, lo stesso arqueiro deve subito dire a quelli che arrivano per il cammino francese: [...]. E ai Lombardi e Toscani deve dire [...]. E a quelli della Tierra de Campos deve dire: [...].

E ciascuna di queste formule si deve pronunciare una volta la mattina, dopo che l'Arca sarà stata menzionata nel perdono, e non prima: dopo questo, egli e gli altri della chiesa devono restare in silenzio mentre viene letta l'indulgenza. E pronunciato il perdono, devono chiamare ogni pellegrino all'Arca in ogni idioma, e devono dire: [...].

E alla fine della mattinata, quando il gruppo di pellegrini si sarà spostato verso l'Altare di San Jacopo, l'arqueiro, o il suo sottoposto che sta li, deve parlare a loro dell'Arca, e se si renderà conto che un pellegrino vuole depositarvi l'offerta per l'Altare del Beato Jacopo, deve dire e mostrargli dove questo si trova, e dirgli che quella [invece] è l'Arca dell'Opera. E i pellegrini devono essere guidati in modo che prima facciano le loro offerte prima all'Altare del Beato Jacopo e poi alla Catena e poi all'Arca dell'Opera e poi agli altri onori.

$$
* * *
$$

Suonata la campana della prima messa, il custode dell'Arca dell'Opera del Beato Jacopo doveva dunque stare in piedi accanto all'Arca per chiamare ad alta voce $\mathrm{i}$ pellegrini e attirarne l'attenzione attraverso dei colpi dati loro sulla schiena (e altrove) con una lunga pertica. Un chierico vestito da messa doveva salire in piedi sull'Arca, e la persona ${ }^{8}$ che elencava e spiegava ad alta voce le varie indulgenze concesse ai pellegrini aveva l'obbligo di menzionare l'Arca prima di ogni altro «onore». Dopo tale menzione, l'arqueiro spiegava agli astanti la differenza tra un'offerta depositata nell'Arca dell'Opera e un'offerta consegnata ai guardiani dell'Altare Maggiore: si trattava di una distinzione tanto importante da obbligare i custodi a comunicarla direttamente alle diverse nazioni di pellegrini nelle loro stesse lingue ${ }^{9}$.

Non è chiaro se si tratta dello stesso arqueiro o di qualcun altro.

L'obbligo di offrire ai pellegrini una spiegazione nel loro proprio idioma andrà probabilmente messo in relazione con un servizio simile descritto quattro secoli più tardi: «El tesoro desta Iglesia son las santas Reliquias que en ella ay, que son muchas, y de gran veneracion, las quales se muestran 
Che la prima nazione a questo punto menzionata sia quella dei Francigeni non desterà alcuna sorpresa. Quello che invece viene come una sorpresa è che la frase che oggi si legge nelle Consuetudines sappia assai più di oc che di oïl:

Ze é l'archa de l'obra mon sennor San Jame, ze é l'obra de la egresa.

Queste parole sembrarono già a Pensado un tentativo da parte di uno scriba galego di produrre un testo occitano: sono conservate le - a finali (archa, obra, egresa) e le vocali toniche brevi e lunghe non dittongano né si frangono (obra $<$ ŎPERA, egresa $<$ ECCLĒSIA), ecc., e gli articoli determinativi conservano la laterale etimologica (l'archa, l'obra, la egresa) (Pensado 1960: 330-332). Quanto al nome attribuito all'Apostolo, San Jame ${ }^{10}$, esso è documentato non solo in testi occitani antichi, ma anche in galegoportoghese: nelle Cantigas de Santa María si parla infatti di un pellegrino che ogni anno si recava «a San Jam'en romaria» (CSM 26.14) e di un uomo di Tolosa che per i suoi peccati ricevette come penitenza di portare un pesante bastone di ferro a Compostela e di collocarlo «ant'o altar de San Jame» (CSM 253.28).

I soli elementi che potrebbero - forse - rinviare alla zona d'oül sono ze e, che potrebbe corrispondere a ce est, ma, come ha mostrato Pensado, potrebbe anche trattarsi di forme limosine oppure - e questo vale anche per il rotacismo della laterale in egresa - doversi a influsso galego (cfr. Pensado 1960: 332, 333).

Ai pellegrini provenienti dall'Italia settentrionale e centrale ${ }^{11}$, l'arqueiro doveva invece dire:

O miçer lombardo, quest'è l'archa dela lavoree de micer sa' Jacome. Questo vay ala gage fayr.

Questa allocuzione parve a Jose Luis Pensado un segno di particolare «respeto e ceremoniosidad con los habitantes de la Peninsula Italiana», non essendo egli al corrente del diffusissimo uso di miçer (-c-/-s-/-ss-) nella penisola iberica per indicare

ciertos dias de la semana a todos los Romeros, por un hombre que para esto está diputado, que sabe de todas lenguas, al qual le llaman el lenguajero» (Molina 1675: 9).

10 López Ferreiro (1902: 65) legge Samanin.

11 L'uso di Lombardia e Toscana per designare genericamente l'Italia del Nord e del Centro è ben documentato nei corpora testuali dell'Istituto Opera del Vocabolario Italiano; $c f r$. due esempi particolarmente chiari: «Ven’ a mostrar gran valore, / pasar Lombardia e Toscana; / in Puglia cotanto vigore / avrà quella viva fontana» (Poes. an. tosc.>ven., 1267, v. 6); «Vate·ne, nova balada, / in Lombardia et in Toschana; / e po' me la va cantando / per la Marcha triv[i]xana» (Poes. an. bergam., 1293, v. 22). 
persone di origine italiana ${ }^{12}$. Va poi notato come per rendere il sintagma latino Archa Operis sia stato fatto ricorso non all'italiano opera, bensì a un sostantivo riconducibile al termine per 'lavoro' nel senso di 'attività fisica', cioè lavorio / -eo / -ero / -iero (dal lat. LABORERIUM) ${ }^{13}$, qui vòlto al femminile, forse per influsso del termine sinonimo e coradicale lavoriera ma ancor più probabilmente a causa del tipo di traduzione, che sarà stata fatta chiedendo a qualcuno i corrispondenti delle singole parole senza considerare il loro valore nella frase ${ }^{14}$.

Più grave la mancanza della parte finale della frase, che avrebbe tra l'altro dovuto segnalare 1'ubicazione dell'Altare Maggiore e spiegare la destinazione delle offerte lasciate nell'uno e nell'altro posto: le parole che seguono al dimostrativo Questo non dànno senso in nessuna delle varietà italoromanze a me note ${ }^{15}$. Supporrei perciò, sebbene con esitazione, un saut du même au même dal brano italiano a un successivo brano in francese (del quale sarebbe andato perso l'inizio): forse qualcosa di simile a «[questo] va à les gages faire» = 'ciò che lasciate qui va a pagare gli stipendi'? Anche qui, però, come già per lavoree, la parola centrale gage compare inspiegabilmente al femminile.

La terza frase in volgare, indirizzata ai Campisinis, cioè, probabilmente, ai pellegrini della comarca detta «Tierra de Campos», è composta in una mescolanza inestricabile di elementi di tipo castigliano (gli articoli determinativi la, las con la laterale conservata, il verbo echar) e tratti galeghi (outra, la mancanza del dittongo in comendas e mortos $)^{16}$. Oltre ai fatti di lingua, mi pare degno di nota il tono complice riservato a questi pellegrini da parte dell' arqueiro, il quale non perde tempo a parlare a loro dell'Altare del Santo, degli «onori» o della Catena, limitandosi a ordinare sbrigativamente che tutti i doni, di qualunque tipo siano, si buttino proprio nell'Arca «e non altrove»:

12 Cfr. Pensado (1960: 335): «nos asombra la fórmula de cortés invocación que inicia la frase, fórmula que falta en los otros casos». Per l'uso di miçer come «blasone» d'italianità nella Spagna del sec. XIII, cfr. Larson (2006: 788-789).

13 Pensado (1960: 337) preferisce pensare a una derivazione lat. LABŌRE(M) > lavore.

14 Questo spiega perché l'articolo sia femminile (era femminile nel modello) e perché si abbia «lavorìo» invece di «opera».

15 Pensado (1960: 338) tenta — purtroppo invano — di dimostrare l'italianità linguistica dell'intero passo: «del examen del texto destinado a orientar la colocación de las ofrendas de los toscanos y lombardos se deduce que está escrito en una lengua mixta, en la que se entremezclan elementos toscanos con otros norteitalianos (lombardos o genoveses)».

16 Di «un pequeno mosaico dialectal» parla Henrique Monteagudo (2004: 61). 
Et vos et del estremo, acá venide a la Archa de la Obra de Señor Santiago; las comendas que trahedes de mortos et de vivos para la Obra de Señor Santiago acá las echade et non en outra parte.

\section{$* * *$}

Nonostante il dovere, più volte ribadito nelle Consuetudines, d'informare i pellegrini sulle due possibili e ben distinte destinazioni delle loro offerte, sono chiaramente i canonici dell'Altare Maggiore ad avere la peggio. Poco importa che nel testo venga precisato che,

...si peregrinus dixerit thesaurariis se portare comendam, thesaurari debent querere ab eo si deffert eam ad Sanctum Jacobum vel ad Archam Operis Sancti Jacobi: et si dixerit se portare per ad Sanctum Jacobum, dicant sibi quod mittat super Altare, et si dixerit se portare ad Archam sive Operam, remittant illum cum ea ad Archam Operis vel ducant illum ad Archam (rr. 28-32)

se un pellegrino dirà ai tesorieri che porta un dono, essi devono chiedergli se lo porta a San Jacopo oppure all'Arca dell'Opera di San Jacopo: e se dirà che lo porta a San Jacopo, essi gli devono dire di collocarlo sopra l'Altare; e se dirà che lo porta all'Arca ossia all'Opera, essi lo devono rimandare, con il suo dono, all'Arca del Opera oppure accompagnarcelo,

perché le raccomandazioni come questa:

debent peregrini ita guiari videlicet quod primitus offerant Altari Beati Jacobi et exinde Cathene et exinde Arche Operis et exinde aliis honoribus (rr. 22-24)

i pellegrini si devono condurre in modo che facciano prima le loro offerte all'Altare del Beato Jacopo, poi alla Catena, e poi agli altri onori

perdono un bel po' della loro forza se si considera che l'arqueiro e i suoi uomini probabilmente non si peritavano di intorbidire le acque, come lascia sottintendere la seguente raccomandazione, senz'altro mèmore di abusi pregressi:

[custodes] debent nominare Archam Operis Beati Jacobi et non Archam Sancti Jacobi, sed Archam Operis Sancti Jacobi (rr. 45-46)

[i custodi] devono parlare dell'Arca dell'Opera del Beato Jacopo e non dell'Arca di San Jacopo, ma proprio dell'Arca dell'Opera di San Jacopo. 
Delle prime tre frasi riportate, soltanto quella rivolta ai Francigenis riferisce ambedue le opzioni (essendo la frase italiana corrotta, non si può escludere che anche in essa si operasse la medesima distinzione), ma con un elemento - la duplice presenza del sostantivo obra - che sembrerebbe fatto apposta per generare incertezza:

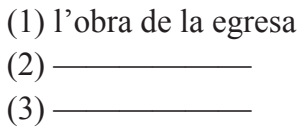

(1) l'obra de la egresa

(3) vs. l'archa de l'obra monseñor San Jame vs. l'archa dela lavoree de micer Sa' Jacome vs. la archa dela obra de Señor Santiago

$* * *$

E così siamo arrivati alla quarta frase alloglotta. A considerare l'impostazione grafica sulla pagina del manoscritto sembrerebbe trattarsi di una lingua pari, quanto a dignità, alle tre precedenti:

[...] ipse arqueyrus debet dicere Francigenis: [...].

I Et Lombardis et Tozcanis debet dicere: [...].

I| Et Campisinis debet dicere: [...].

ๆ Et debet dicere: [...].

Tuttavia la proposta di Pensado di vedere in btom un riferimento a una lingua poco nota ma pur sempre reale come il bretone ${ }^{17}$ presuppone una sostanziale simmetria tra le quattro frasi, laddove invece ci aspetteremmo che l'ultima fosse di solennità più marcata: dopo tutto era soltanto dopo avere assistito in silenzio alla lettura pubblica della formula di indulgenza che si doveva vocare totum peregrinum ad Archam per totam linguaginem e dire quello che si doveva dire.

Per trovare un'interpretazione autorevole della frase misteriosa, López Ferreiro si era rivolto a un erudito con fama di grande archeologo ed epigrafista, il gesuita catalano Fidel Fita y Colomé (1835-1918), ricevendone una risposta che ancor oggi conta qualche sostenitore:

17 «[N]os atrevemos a proponer la lección: 'debet dicere bretoni' con lo que naturalmente las palabras siguientes estarán en bretón» (Pensado 1960: 340). Colgo l'occasione per aggiungere che l'amico celtista Andrea Nuti dell'Università di Pisa mi ha assicurato della non-celticità del passo in discussione. Per la percezione del bretone come lingua per definizione incomprensibile, ricordo che nella famosa scena della quattrocentesca Farce de Maistre Pierre Pathelin in cui l'omonimo protagonista vaneggia in numerose lingue per sembrare moribondo agli occhi di un creditore, quando, dopo aver parlato limosino, piccardo, normanno e fiammingo, il finto malato arriva al bretone, il suo creditore si arrende e dichiara: «Il ne parle pas crestien / Ne nul langaige qui apere» ['Non parla cristiano, né alcuna lingua che si riconosca']: vedi Schneegans (1908: vv. 929-930). 
[...] pronunciábase por el sacerdote la fórmula de la absolución, y llamando en seguida á los peregrinos, según la lengua de cada uno, decía, dirigiéndose al santo Apóstol, estas palabras: Betom a atrom San Giama! A atrom de labro, como si dijera, según la interpretación del R. P. Fita: Bien toma el trueno, Santiago! El trueno del labio, ó lo que es lo mismo: «Recibe benignamente, Apóstol Santo, este grito atronador que en todas las lenguas del mundo pronuncia el labio.» El sabio Jesuita cree que aquella frase está en gallego puro. «Ninguna otra lengua, dice en carta á nuestro amigo D. Antonio López Ferreiro, da este sentido, y además se trata del concurso ó se habla por los eclesiásticos á totum peregrinum per totam linguaginem. Es, concluye, Galicia gloriosa que ruega á su Apóstol acepte la ofrenda de todas las lenguas.» ${ }^{18}$.

Ovviamente non è possibile dare credito all'ipotesi del padre Fita: ce lo vieta, se non altro, la grammatica storica, giacché in nessuna fase della sua lunga esistenza la lingua galega avrebbe potuto produrre un enunciato come quello.

Esiste una possibilità di arrivare a un'interpretazione convincente? Forse sì, ma trattandosi di una lingua totalmente sconosciuta dobbiamo procedere con molta cautela. Partendo dalla trascrizione diplomatica btom aatrō sangyama aatrō d'labro notiamo in primo luogo che i compendi del primo, secondo e quarto gruppo grafico si potrebbero sciogliere in più di un modo, rispettivamente bertom / bartom / bretom e aatrom / aatron; il quinto gruppo permette invece soltanto delabro.

Mettendoci nei panni di un lettore di ambito chiesastico del secolo XIII, abituato quantomeno ad affrontare testi in latino, galego e castigliano, le unità lessicali apparenti potrebbero essere le seguenti: «btom a atrō san gyama a atrō del abro». Questo non ci porta molto lontano, ma almeno ha l'aria di una lingua vera; se poi, avendo tentato invano varie permutazioni di elementi, proviamo semplicemente a invertire l'ordine delle vocali nelle singole unità, otteniamo questo:

btom a ōtra [oppure otrā?] san gyama a ōtra [oppure otrā?] del obra.

18 Fernández Sanchez \& Freire Barreiro (1885: 38). L'autorità del gesuita catalano è rimasta forte al di sopra di ogni commento critico praticamente fino ai giorni nostri, come mostra per esempio la citazione che ne fa Ramón Otero Pedrayo nella sua Guía de Santiago de Compostela: «La peregrinación en nuestros años vuelve a tomar la grandeza y devoción de los buenos tiempos. Es el mismo consuelo y rumor de himnos de cuando el sacerdote después de absolver al peregrino exclamaba dirigiéndose a la imagen del Apóstol: ‘Be tom a trom Sangiama! ¡Atrom de labro! Recibe benignamente este grito atronador que en todas las lenguas de la tierra pronuncia el labio!', según la interpretación del P. Fita. Es la voz de la variada cristiandad sonando conjunta en el ámbito del templo de Santiago, con palabras del romance gallego» (Otero Pedrayo 1945: 27). 
Non è né galego né castigliano, ma si lascia leggere: il parallelismo tra il primo e il secondo ôtra mostra san gyama contrapposto a del obra. Questo, combinato con i due a ôtra (o otrā), se interpretati come una costruzione del tipo lat. ALTER ... ALTER ['il primo ... il secondo'], finisce per conferire alla frase un significato complessivo analogo a quello delle tre precedenti: 'l'una è per san Jacopo, 1'altra è dell'Opera'19.

Allora il misterioso idioma sarebbe servito soltanto a confermare il messaggio già espresso in tre (o magari quattro, se è giusta la mia ipotesi su vay ala gage fayr) lingue esistenti? Forse sì: anche se non si capisce la ragione per cui una lingua inventata - o che sembrerebbe essere tale — sia stata usata per trasmettere informazioni indispensabili. Le ipotesi sull'origine della frase potrebbero essere tante: un'aggiunta marginale di difficile lettura, magari una glossa, copiata acriticamente nel testo? Oppure, data l'impossibilità di esprimere per totam linguaginem i concetti richiesti, lo scrittore avrà deciso di adoperare una lingua che non esisteva e che quindi poteva rappresentarle tutte? Purtroppo sono spiegazioni che lasciano un po' il tempo che trovano.

Il mio netto rifiuto dell' interpretazione (para-)liturgica del padre Fita deriva, come già detto, da fatti linguistici, ma soprattutto dalla consapevolezza, maturata attraverso una pluriennale frequentazione di archivi ecclesiastici e monasteriali in Italia, Spagna e altrove, che nei registri di parrocchie, capitoli cattedralizi, diocesi, ecc., le questioni spirituali sono nettamente separate da ciò che invece riguarda i beni terreni. Sarebbe stato poco meno che assurdo se, nel resoconto di un' inchiesta sul modo in cui i chierici dell'Altare Maggiore e i laici dell'Arca dell'Opera si spartivano le offerte monetarie e in natura lasciate dai pellegrini, fossero state inserite preghiere, formule di benedizione o giaculatorie. I chierici e i laici fabbricieri del sec. XIII sapevano bene che non è possibile servire allo stesso tempo Dio e Mammona.

\section{BIBLIOGRAFIA}

Fernández Sanchez, J. M. \& Freire Barreiro F. (1885): Guia de Santiago y sus Alrededores. Santiago: Imprenta del Seminario Conciliar.

LARSON P. (2006): «Ço es amors e altre possibili tracce italiane in poesia occitanica del secolo XIII», in P. G. Beltrami \& M. G. Capusso \& F. Cigni \& S. Vatteroni

\footnotetext{
19 La prima parola, bertom, ecc., resiste strenuamente a ogni mio tentativo di decifrazione (a meno che non si voglia pensare a un qualche nesso con il verbo vertere o con l'avverbio basco berton 'aqui mismo').
} 
(a cura di), Studi di Filologia romanza offerti a Valeria Bertolucci Pizzorusso. Pisa: Pacini Editore, pp. 777-803.

López Ferreiro, A. (1902): Historia de la Santa A. M. Iglesia de Santiago de Compostela. III. Santiago: Imprenta y Encuadernación del Seminario Conciliar Central.

Molina, B. S. (1675): Descripcion del Reyno de Galicia y de las cosas notables del

[...] Compuesto por el Licenciado Molina. Madrid: Por Roque Rico de Miranda. Monteagudo, H. (2004): «O Camiño, as linguas e a emerxencia do galego», Grial 161, pp. 52-61.

Otero Pedrayo, R. (1945): Guía de Santiago de Compostela. Santiago de Compostela: Editorial Compostela.

Pensado, J. L. (1960). «Aspectos lingüísticos de la Compostela medieval», Vox romanica 19 , pp. 319-340.

Pérez Rodríguez, F. J. (2004): Os documentos do Tombo de Toxos Outos. Santiago de Compostela: Consello da Cultura Galega.

SchneEgans, F.-E. (1908): Maistre Pierre Pathelin, farce du XVe siècle. Strasbourg: Heitz.

VÁzQuez de PARga, L. \& LACARRA, J. M. \& URÍA RÍU, J. (1948-49): Las peregrinaciones a Santiago de Compostela. Madrid: C.S.I.C. 